\title{
El movimiento por la planificación familiar en Murcia y el acceso de las mujeres a la anticoncepción, 1976-1980
}

\author{
The Family Planning Movement in Murcia and the Access \\ of Women to Contraception, 1976-1980
}

\author{
Ramón CASTEJóN BoLEa \\ Universidad Miguel Hernández *
}

\begin{abstract}
Resumen: Durante la década de 1970 se constituyó en España un movimiento social por la planificación familiar. En este proceso confluyeron intereses sociales, políticos y científico-sanitarios cuyos resultados fueron la despenalización de los anticonceptivos, prohibidos en España desde 1941, y el desarrollo de infraestructuras que facilitaran su accesibilidad a todas las mujeres. Una de las características de este proceso fue la diversidad regional de modelos en el origen, desarrollo y consolidación de los centros de planificación familiar. La investigación que se propone pretende describir y analizar el caso de la Región de Murcia como ejemplo de este proceso en la periferia española, con sus singularidades y similitudes en el contexto nacional. El estudio se centra en el periodo 1976-1980, dentro de la primera fase del movimiento de planificación familiar en nuestro país cuando se consigue la legalización de todos los métodos anticonceptivos.
\end{abstract}

Palabras clave: Sexualidad; anticoncepción; feminismo; Región de Murcia.

\begin{abstract}
During the 1970's a social movement for family planning began in Spain. Social, political and health science interests came together, resulting in the decriminalization of contraceptives which had been forbidden in Spain since 1941, and in the development of infrastructures which facilitated the access of all women to them. One aspect that characterized this process was the regional diversity of the models regarding the origin, development and consolidation of the family planning centers. The proposed research seeks to describe and analyze the case of the Region of Murcia as an example of this process in the Spanish periphery, with its singularities and similarities within the national context. The study focuses on the period between 1976 and 1980 within the first phase of the family planning movement in our country, when the legalization of all contraceptive methods is achieved.
\end{abstract}

Key words: Sexuality; Contraception; Feminism; Region of Murcia.

* Recibido: 15, marzo, 2017. Aceptado: 8, mayo, 2018.

Área de Historia de la Ciencia, Universidad Miguel Hernández. Este trabajo ha sido desarrollado en el marco del proyecto de investigación ASYS: Anticoncepción, sexualidad y salud durante el Franquismo y la transición a la democracia (HAR2012-39644-C02-01), financiado por el Ministerio de Economía. Email: rcastejonb@umh.es. 


\section{INTRODUCCIÓN}

En 1971 un Comité de Expertos de la Organización Mundial de la Salud (OMS) redactó un informe en el que definió el término Planificación Familiar (PF), recomendando a todos los gobiernos su inclusión en los servicios sanitarios. ${ }^{1}$ Los objetivos de estos servicios debían ser: respetar los derechos humanos de individuos, parejas y familias; regular el crecimiento demográfico de la población y mejorar la salud de la familia y de la comunidad. Efectivamente, a partir de la década de los setenta del siglo pasado, en todos los países industrializados, el acceso a la anticoncepción empezó a formar parte de los sistemas públicos o privados de atención sanitaria. El activismo en favor de la anticoncepción comenzó en la década de 1920 en países de Europa y Estados Unidos con el movimiento neomalthusiano y feminista bajo la denominación de birth control. ${ }^{2}$

En nuestro país, durante la década de 1970 se constituyó un movimiento social muy activo por la planificación familiar. Los objetivos de este movimiento eran la despenalización de los anticonceptivos, prohibidos en España desde 1941, y el desarrollo de infraestructuras que facilitaran su accesibilidad a todas las mujeres. Una de las características de este proceso fue la diversidad regional de modelos en el origen, desarrollo y consolidación de los centros de planificación familiar así como las diferentes interacciones entre los intereses de carácter social y político-feministas, democráticos, pro-derechos humanos-y científico-sanitarios que confluyeron en el proceso. La legalización de la venta y divulgación de información sobre los anticonceptivos fue uno de los temas importantes del debate social y político de la transición democrática. De hecho, el proceso de la despenalización de los anticonceptivos, iniciado en la primavera de 1978 y concluido en diciembre de ese año, fue una de las primeras reformas del Código Penal de las cortes democráticas. ${ }^{3}$

1 INFORME DEL COMITÉ DE EXPERTOS DE LA OMS: La planificación de la familia en la acción sanitaria, Serie de Informes Técnicos n. 417, Ginebra: Organización Mundial de la Salud, 1971.

2 ORTIZ GÓMEZ, T.; RODRIGUEZ OCAÑA, E. y GIL, E.: «Políticas anticonceptivas y ciudadanía en España, del franquismo a la monarquía parlamentaria», comunicación no publicada presentada al X Congreso de la Asociación de Demografía Histórica, Albacete, del 18 al 21 de junio de 2013. Para un acercamiento a las complejas interacciones entre ciencia, medicina y feminismo en lo relativo a la anticoncepción, v. ORTIZ-GÓMEZ, T.: «Maternidad voluntaria: Anticoncepción, ciencia y feminismo en el siglo XX», en G. Franco (ed.), Debates sobre la maternidad. Desde una perspectiva histórica (siglos XVI-XX), Barcelona: Icaria, 2010, pp. 85-105.

3 IGNACIUK, Á.: La Anticoncepción Hormonal en Polonia y España. Discursos, debates y prácticas entre 1960 y 1980, Tesis Doctoral, Universidad de Granada, 2015, p. 97. 
Como han señalado Teresa Ortiz-Gómez y Ágata Ignaciuk, los principios del activismo por la planificación familiar en España se identificaban en la literatura existente con el activismo feminista por el derecho a la anticoncepción y al aborto durante la segunda mitad de la década de $1970 .{ }^{4}$ Sin embargo, y de manera similar a lo ocurrido en Italia y Francia, el activismo por la planificación familiar en España se originó inicialmente en círculos médicos a finales de la década de 1960, antes de expandirse significativamente en la siguiente década gracias al compromiso de las organizaciones de mujeres radicales y su colaboración con los profesionales médicos liberales.

El movimiento de planificación familiar en nuestro país tuvo dos fases: una que alcanza hasta 1980, cuando se consigue la legalización de todos los métodos anticonceptivos con excepción del aborto, y otra limitada a los años 1980-1985, centrado en la expansión de una nueva forma de asistencia sanitaria privada y pública en forma de consultas y centros de planificación familiar y en el debate y el activismo por la despenalización del aborto. ${ }^{5}$

La investigación que se pretende realizar se sitúa en la sociedad murciana durante el tardofranquismo y la transición democrática. De manera especial nos interesa el protagonismo de los movimientos sociales y la sociedad civil durante el proceso histórico de la transición política y el de la descentralización territorial, que se pone en marcha en la región a partir de 1978. Hay que recordar que la sociedad murciana que va a enfrentar estos procesos de transformación política, social y cultural está condicionada por su retraso en el proceso de modernización.

4 FERREIRA, S. L.: «El Movimiento Feminista y la Salud de las Mujeres: La Experiencia de los Centros de Planificación Familiar (Cpf) en Catalunya (1976-1982)», Revista Estudios Feministas, 16, n. 3, 2008, pp. 785-807; MERRÉ BALDRICH, M.: «Los Centros de Planificación Familiar (Cpf) en Cataluña: Logro del Movimiento Feminista en la Transición», en A. González González (ed.): No es País para Jóvenes. Encuentro de Jóvenes Investigadores en Historia Contemporánea, Vitoria: Instituto Valentín de Foronda, 2012, pp. 1-21, citado en: ORTIZ-GÓMEZ, T. \& IGNACIUK, Á.: «The fight for family planning in Spain during late Francoism and the transition to democracy, 1965-1979», Journal of Women History, 32, n. 2, 2018, pp. 38-62.

5 ORTIZ GÓMEZ, T.; FAJARDO, A.; GIL GARCÍA, E.; IGNACIUK, Á. y RODRÍGUEZ OCAÑA, E.: «Activismo feminista y movimiento asociativo por la planificación familiar en España», en M. I. Porras Gallo; B. Gutiérrez Rodilla; M. Ayarzagüena Sanz y J. de las Heras Salord (eds.): Transmisión del conocimiento médico e internacionalización de las prácticas sanitarias: una reflexión histórica. XV Congreso SEHM, Madrid: SEHM-Facultad de Medicina de la UCAM, 2011, pp. 141-145. GIL GARCÍA, E.; ORTIZ GÓMEZ, T. \& IGNACIUK, Á.: «El movimiento de planificación familiar en la ciudad de Sevilla durante la Transición Democrática (1975-1983)», en I. Vázquez Bermúdez (coord.), Investigación y Género. Logros y retos, Sevilla: Universidad de Sevilla, 2011, pp. 726-736. 
La denominada transición democrática, es un proceso histórico que ha sido objeto de distintas y diversas interpretaciones. Resumiendo, podemos hablar de tres modelos explicativos: el que pone el énfasis en los cambios sociales y económicos habidos en la España de los años sesenta; el que presenta el proceso como la obra de una élite política formada, sobre todo, por los reformistas del régimen con el nuevo monarca al frente $y$, finalmente, los que destacan el protagonismo de los movimientos sociales, sobre todo los sindicatos obreros, las organizaciones estudiantiles universitarias y las asociaciones de vecinos, en la pérdida de legitimidad y capacidad de supervivencia del régimen franquista. ${ }^{6}$

Utilizando entrevistas orales con profesionales del ámbito de la planificación familiar y activistas feministas de Murcia, así como prensa generalista de la Región, la investigación que se propone pretende describir y analizar el caso de la Región de Murcia entre 1976 y 1980 como ejemplo en la periferia española de este proceso de activismo y desarrollo de la planificación familiar, con sus singularidades y similitudes en el contexto nacional.

\section{UNA SOCIEDAD RURAL / TRADICIONAL EN TRANSFORMACIÓN: LA TRANSICIÓN DEMOCRÁTICA Y EL PROCESO DE DESCENTRA- LIZACIÓN TERRITORIAL EN MURCIA}

Durante la segunda mitad del siglo XX el aumento de la población en Murcia fue más elevado que el del conjunto español: entre 1941 y 1985 la tasa de crecimiento de población en Murcia duplicó la de España. Este aumento se produjo a pesar del fuerte movimiento migratorio, que ocurrió especialmente en la década de 1960 a 1970, y fue provocado por las altas tasas de natalidad y nupcialidad propias de una población más joven que la del resto del país. ${ }^{7}$ Por su parte, la tasa bruta de natalidad en la provincia de Murcia desde 1950 hasta 1992 fue superior al resto de España, aunque en 1984 el índice de fecun-

6 QUIROSA-CHEYROUZE y MUÑOZ, R.: «Transición a la democracia: una perspectiva historiográfica», en R. Quirosa-Cheyrouze y Muñoz (coord.): Historia de la Transición en España. Los inicios del proceso democratizador, Madrid: Biblioteca Nueva, 2007, pp. 13-27. Una síntesis de los dos primeros modelos explicativos en JULIÁ, S.: «Cambio social y cultura política en la transición a la democracia», en J. C. Mainer y S. Juliá: El aprendizaje de la libertad, 1973-1986, Madrid: Alianza Editorial, 2000, pp. 15-77.

7 PÉREZ PICAZO, M. T.: «Una reflexión sobre la historia de la región murciana. Progresos e inconsecuencias de las investigaciones en las últimas décadas», en E. Nicolás y J. A. Gómez (coords.): Miradas a la Historia. Reflexiones historiográficas en recuerdo a Miguel Rodríguez Llopis, Murcia: Universidad de Murcia, 2004, pp. 101-127 (p. 117). A partir de 1978, la Región de Murcia se convierte en una región con saldo migratorio positivo. 
didad ya fue inferior a 2,1 hijos por mujer (índice de relevo generacional). El número de hijos por mujer en edad fértil había pasado de 3,3 en 1975 a 2,9 en 1980 y continuó descendiendo hasta los 2,0 en $1985 .{ }^{8}$ En cuanto a la distribución territorial, no sería hasta los años 60 cuando empieza a configurarse una población urbana que acabará sobrepasando a la rural. En la década de 1970 por primera vez en su historia, la población urbana supera a la rural. En 1981, Murcia, Cartagena y Lorca tenían ya más del $50 \%$ de toda la población de la región.

Las décadas de 1960-1970 supusieron para la región de Murcia una aceleración del proceso de modernización interrumpido durante la primera etapa del franquismo. Entre 1964 y 1975 la economía regional creció a una tasa anual media cercana al 7\%, situándose entre las regiones que experimentaron un crecimiento más fuerte. Sin embargo, en pleno desarrollismo, la región presentaba uno de los niveles más altos de desigualdad en el conjunto de las regiones y continuaba situada en la España pobre. En la década de los setenta la población agraria había descendido ( $23,9 \%$ de la población activa) y se produjo un crecimiento significativo de los servicios $(39,4 \%)$, además de un lento pero firme proceso de industrialización (25\%). ${ }^{9}$ Por su parte, los valores y pautas de consumo o de ocio que han predominado ampliamente en Murcia a lo largo de los siglos XIX y XX han sido los característicos del mundo campesino. ${ }^{10}$ Durante las décadas de los 50 y 60 la ciudad de Murcia era una ciudad relativamente pequeña con una sociedad tradicional y predominantemente conservadora, en la que las relaciones sociales y la moral estaban fuertemente influenciadas por el franquismo y la Iglesia Católica. ${ }^{11}$

Como ha estudiado la historiografía contemporánea, durante los años 1974, 1975 y 1976 la conflictividad social aumentó en Murcia. Huelgas, manifestaciones y encierros se suceden estos años en la provincia y la capital. Durante 1976 se producen en la provincia múltiples conflictos sociales, laborales y en el mundo de

8 FRUTOS BALIBREA, L. y MELLADO CARRILLO, M. (coords.): Estructura y cambio social en la Región de Murcia. Vol. I. Demografía, Estructura Productiva y Mercado Laboral en la Región de Murcia, Murcia: Universidad de Murcia, 1996, pp. 28, 38-39. En 1975 la tasa de natalidad por 1.000 habitantes era de 22,3, en 1980 de 19,8 y en 1985 de 15,0. En el 2005, la tasa de natalidad era de $12,97 \%$ habitantes. Los extranjeros aportaban ya un $20 \%$ a esta natalidad.

9 MARTÍNEZ CARRIÓN, J. M.: Historia económica de la Región de Murcia. Siglos XIX y XX, Murcia: Editora Regional, 2002, pp. 478-491.

10 PÉREZ PICAZO, M. T., 2004, pp. 101-127.

11 NICOLÁS MARÍN, M. E.: «Murcia durante la dictadura de Franco (1939-1975)», en M. E. Nicolás (coord.): Historia contemporánea de la Región de Murcia, Murcia: Ediciones de la Universidad de Murcia, 2014, pp. 265-314. 
la enseñanza. ${ }^{12}$ Sin embargo, el éxito de la reforma política en Murcia se refleja en los resultados del referéndum del 15 de diciembre de ese año convocado por el gobierno de Adolfo Suárez, con una participación del 82\% de los electores y con el $95 \%$ de los votos a favor. ${ }^{13}$ Votaron el $77,8 \%$ de los españoles y a favor un 94,17\%. La movilización social y política en Murcia continuaría durante finales de los años setenta y principios de los ochenta, fundamentalmente liderada por el movimiento obrero y vecinal. Muchos de los líderes de estos dos movimientos se incorporarán, a partir de las elecciones municipales de 1979, a los primeros ayuntamientos democráticos, sobre todo en las listas socialistas (PSOE) y comunistas (PCE), ${ }^{14}$ y también de otros partidos.

El 1 de marzo de 1979, primeras elecciones legislativas tras el periodo constituyente de las Cortes Generales, y 3 de abril de 1979, primeras elecciones locales, se produjo en Murcia el cambio político de la Unión de Centro Democrático (UCD) al PSOE, que pasó a ser mayoritario en todas las instituciones regionales murcianas desde 1979. ${ }^{15}$ Por su parte, en 1978 se puso en marcha el régimen preautonómico -el Real-Decreto Ley de 27 de septiembre de 1978 aprobó el régimen preautonómico para la región de Murcia-y el 25 de mayo de 1982 el Congreso de los Diputados aprobó el Estatuto de Autonomía de la Región de Murcia. La nueva institucionalidad democrática de naturaleza autonómica, puesta en marcha con el Consejo Regional de Murcia, se constituyó el 10 de noviembre de 1978, con Antonio Pérez Crespo (UCD) como primer presidente del ente pre-autonómico. Un año más tarde, en mayo de 1979, el nuevo Consejo Regional de Murcia estaría ya presidido por el socialista Andrés Hernández Ros. ${ }^{16}$

12 GONZÁLEZ MARTÍNEZ, C.: «Viejo y nuevo antifranquismo en Murcia», en A. Mateos López y Á. Herrerín López (coords.): La España del presente: de la dictadura a la democracia, Madrid: Asociación Historiadores del Presente, 2006, pp. 59-82; NICOLÁS MARÍN, M. E. y GONZÁLEZ MARTÍNEZ, C.: «Caminos de libertad (I): Movimientos sociales y disidencia juvenil», en M. E. Nicolás Marín (coord.): Historia contemporánea de la Región de Murcia, 2014, pp. 349-379.

13 NICOLÁS MARÍN, M. E.: «La Transición se hizo en los pueblos. La vida política en Murcia (1968-1977)», en R. Quirosa-Cheyrouze y Muñoz (coord.): Historia de la Transición en España. Los inicios del proceso democratizador, 2007, pp. 251-268.

14 GONZÁLEZ MARTÍNEZ, C.: «El tránsito de la dictadura a la democracia en Murcia. Acción colectiva, respuestas institucionales y posicionamientos políticos», Ayer, 79, n. 3, 2010, pp. 87-120.

15 Véase una síntesis de las elecciones democráticas en Murcia durante la transición en: GONZÁLEZ MARTÍNEZ, C. y NICOLÁS MARÍN, M. E.: «Caminos de Libertad (II). Elecciones y práctica democrática en las instituciones. Olvidos y memorias de la Transición», en M. E. Nicolás Marín (coord.): Historia contemporánea de la Región de Murcia, 2014, pp. 381-410.

16 Ibídem; GONZÁLEZ MARTÍNEZ, C. y ESCUDERO ANDÚJAR, F.: «La Transición en Murcia. Movimientos sociales y poder político. Nuevas perspectivas de investigación», en R. QuirosaCheyrouze y Muñoz (coord.): La sociedad española en la Transición. Los movimientos sociales en el proceso democratizador, Madrid: Biblioteca Nueva, 2011, pp. 383-399. 


\section{EL DERECHO A LA ANTICONCEPCIÓN Y EL MOVIMIENTO FEMI- NISTA EN MURCIA}

Durante el tardofranquismo y la transición a la democracia, los movimientos sociales de la Región de Murcia jugaron un papel esencial en la conquista de libertades fundamentales. Al igual que en el resto del país, la sociedad civil aprovechó el nuevo marco asociativo instaurado por la Ley de Asociaciones de 1964. La actividad de las asociaciones en el proceso de transición a la democracia aumentó gradual y extraordinariamente a lo largo de la década de 1970, especialmente en las actividades de las asociaciones de vecinos y en las asociaciones de padres de alumnos. Éstas tuvieron en Murcia un gran protagonismo en las reivindicaciones sociales y culturales, y fueron encauzando la acción social hacia la actuación política. ${ }^{17}$ Así, el movimiento social concretado en las asociaciones de vecinos fue politizando sus demandas en el transcurso de los primeros años de la década de los $70 .{ }^{18}$ En el ámbito de la salud, las asociaciones de vecinos van a canalizar gran parte de las demandas ciudadanas en materia de asistencia sanitaria y salud pública en la región. ${ }^{19}$

No hay, que conozcamos, ninguna monografía dedicada específicamente a las organizaciones de mujeres y feministas en la Región de Murcia, ${ }^{20}$ por lo que utilizaremos los trabajos donde se explora este movimiento social. ${ }^{21}$ Se considera que la primera organización de mujeres en la década de los 70 en Murcia fue el Movimiento Democrático de Mujeres (MDM), fundado en Murcia en 1973 por Elvira Ramos, Nieves Fernández y Ana Martín, todas ellas miembros del PCE en la clandestinidad. Según Marín Gómez, «de la lectura de prensa, especialmente

17 MARÍN GÓMEZ, I.: Asociacionismo, sociabilidad y movimientos sociales en el franquismo y la Transición a la democracia. Murcia, 1964-1986, Tesis Doctoral, Universidad de Murcia, 2007, pp. 143 y 144.

18 GONZÁLEZ MARTÍNEZ, C., 2006, p. 80.

19 MARÍN GÓMEZ, I., 2007, p. 419.

20 Hay que mencionar un trabajo sobre el asociacionismo de mujeres en los centros de la mujer del municipio de Murcia impulsado por el Ayuntamiento, con mayoría socialista durante la década de los 80. ALEMÁN PÉREZ, S.: El origen de los Centros de la Mujer en el municipio de Murcia, Alzira: Germania, 2006, pp. 89-111.

21 NICOLÁS MARÍN, M. E. y GONZÁLEZ MARTÍNEZ, C., 2014, pp. 349-379; MARÍN GÓMEZ, I., 2007, pp. 520-528. Para un acercamiento al movimiento feminista en España de mediados de los 70 y su interés en temas de cuerpo y salud de las mujeres, véase NASH, M.: Dones en Transició. De la Resistència Política a la Legitimat Feminista: Les Dones en la Barcelona de la Transició, Barcelona: Ajuntament de Barcelona, 2007 y THRELFALL, M.: «Feminist Politics and Social Change in Spain», en M. Threlfall (ed.): Mapping the Women's Movement: Feminist Politics and Social Transformation in the North, London: Verso, 1996, pp. 115-151. 
entre 1974 y 1989, se desprende la extraordinaria actividad pública que llevaba a cabo dicha organización femenina». ${ }^{22}$ Sin embargo, entre sus propuestas reivindicativas iniciales en Murcia no figuraba el derecho a la anticoncepción. ${ }^{23}$ Esta posición cambiaría posteriormente. ${ }^{24}$

A esta organización siguieron, según Marín Gómez, la Asociación Cultural de Mujeres Feministas de Murcia (1985), el Colectivo Feminista de Murcia (1985), el Movimiento de Mujeres de Murcia (1985), el Colectivo Progresista de Mujeres de Cartagena (1985), el Colectivo Feminista de Cartagena (1986) y el Colectivo Feminista de Molina (1986). Todas ellas fueron registradas como asociaciones. No se refiere, sin embargo, a la Asamblea de Mujeres de Murcia, una organización feminista asamblearia y autónoma de los partidos políticos que inició su andadura en torno a 1978-1979 en Murcia. ${ }^{25}$ La Asamblea de Mujeres de la Región de Murcia se creó, según dos de sus activistas, Ángeles Muñoz y Reyes Naval, en mayo de $1978 .{ }^{26} \mathrm{Al}$ parecer de una de sus activistas, ésta fue la primera organización feminista en la ciudad de Murcia: «Que yo conocí, creo que sí. Que tenía organización, que tenía estructura, que tenía pensamiento propio, que tenía vinculación y coordinación con el movimiento feminista. Yo creo que sí. Yo creo que sí, que fue la primera». ${ }^{27}$

Aglutinaba en marzo de 1980 a medio centenar de mujeres y se definía como un movimiento autónomo de los partidos políticos aunque algunas de sus componentes militaban en algún partido político de la izquierda y extrema izquierda. $\mathrm{Su}$ actividad se mantuvo entre 1978 y 1982 . Desde su nacimiento estuvo presente el debate de la doble militancia:

22 MARÍN GÓMEZ, I., 2007, p. 524. Para esta organización, v. ARRIERO RANZ, F.: El Movimiento Democrático de Mujeres. De la lucha contra el franquismo al feminismo (1965-1985), Madrid: Los Libros de la Catarata, 2016.

23 MARÍN GÓMEZ, I., 2007, pp. 524-525. Al enumerar los fines de la Asociación de Madres Trabajadoras de Yecla -inscrita en el Registro de Asociaciones en 1978- no incluye esta reivindicación. Según esta autora los fines de esta asociación coincidían con las reivindicaciones del MDM. Algunas autoras han señalado el escaso interés del MDM por los temas de salud de las mujeres y la planificación familiar hasta mediados de los 70. ORTIZ-GÓMEZ, T. \& IGNACIUK, Á.: «The fight for family planning in Spain...», 2018.

24 ARRIERO RANZ, F., 2016, pp. 284-285: «El contacto de las activistas del MDM con la segunda ola del feminismo fue para algunas una experiencia transformadora, debido entre otras cuestiones, a los retos que planteaba hacer de lo personal un asunto político o redescubrir el cuerpo y la sexualidad. Para otras, esas ideas les llevaban a un cuestionamiento global de sus vidas y las rechazaron».

25 Esta organización es comentada en ALEMÁN PÉREZ, S., 2006, pp. 95-111.

26 «Dos mil mujeres esperan consulta sobre planificación familiar en la Arrixaca», Línea, 9 de marzo de 1980.

27 Ana Mula, feminista, bióloga, militante del Movimiento Comunista, entrevistada por Ramón Castejón, Murcia, 7 de octubre de 2016. 
«Había ahí el debate de la doble militancia o de la militancia en el movimiento feminista. Los partidos entonces, pues tipo el MC, la Liga Comunista, incluso pues yo creo que el Partido Socialista o el Partido Comunista, pues éramos un poco, un poco no, éramos partidarios de la doble militancia... Pero esa doble militancia también pasaba por tener una separación. No se entendía que las mujeres pudiéramos entender, liderar el movimiento feminista, haciéndolo siempre con los hombres. Entonces las mujeres necesitaban también la autonomía. Esa autonomía. Entonces la Asamblea de Mujeres y muchos movimientos feministas se alimentó de mujeres que militaban en otros partidos políticos, pero que entendían que en eso se necesitaba autonomía. Se necesitaba romper la dependencia que había con los hombres incluso en los partidos que nos creíamos que éramos los más progresistas y los más modernos del mundo. Entonces ahí surgió la Asamblea de Mujeres. Y la Asamblea de Mujeres, en la Asamblea de Mujeres había una confluencia de mujeres que venían del PSOE, del PC, de la Liga, del Movimien..., y mujeres independientes. De hecho también había muchas mujeres, pues que nos recriminaban un poco que nuestra ideología no era tan puramente feminista o tan independiente como debiera ser porque de alguna manera teníamos que compaginarla y casarla con los postulados de los partidos políticos».28

A finales de los años 70 y primeros de los 80, por tanto, la Asamblea de Mujeres de Murcia se constituye probablemente en la organización feminista más importante de la ciudad:

«Entonces así fue como surgió la Asamblea de Mujeres. Y aglutinaba, la verdad es que en eso yo creo que tuvo un valor importante, que era bastante unitaria, aglutinaba a todas las mujeres que nos movíamos entonces, por lo menos aquí en Murcia, militando en los partidos políticos. Que éramos muchas estudiantes, algunas trabajadoras y otras profesionales jóvenes. Profesionales que ya nos llevaban unos años, habían acabado sus carreras y estaban trabajando». 29

La organización tenía varias comisiones o grupos de trabajo dedicados a diferentes temas: trabajo, educación, propaganda... Uno de los grupos de trabajo se centraba en el acceso a la anticoncepción -recordemos que se legaliza en 1978-, en el derecho al aborto «libre y gratuito» y en la sexualidad de las mujeres. Estos dos últimos temas eran centrales en la agenda del feminismo. ${ }^{30} \mathrm{De}$

28 Ibídem.

29 Ibídem. Marín Gómez considera que «Los movimientos feministas y ecologistas, a través del asociacionismo, no serán excesivamente fuertes en Murcia. El primero fue más asumido por el MDM, impulsado por las mujeres del PCE, que por los colectivos feministas que se constituyen en asociación». MARÍN GÓMEZ, I., 2007, p. 557.

30 ORTIZ GÓMEZ, T.: «Conocer el propio cuerpo para acabar con el patriarcado. Publicaciones feministas sobre salud en España durante la transición democrática», en R. Campos Marín; Á. González de Pablo; M. I. Porras Gallo y L. Montiel: Medicina y poder político. XVI Congreso SEHM, Madrid: SEHM-Facultad de Medicina de la UCM, 2014, pp. 259-263. 
hecho, en 1979, la Asamblea de Mujeres presentó al ente preautonómico un proyecto de centro de información y asesoramiento de la mujer en materia psicológica, jurídica, cultural y sexual. ${ }^{31}$

El otro movimiento social donde el activismo de las mujeres se desarrolló y que también se hizo eco de la lucha por el derecho a la anticoncepción fue el movimiento vecinal. A modo de ejemplo, antes de la legalización, en junio de 1977, un grupo de mujeres de Cieza, vinculadas al movimiento vecinal, organizaban en el salón parroquial del barrio de San José Obrero un ciclo de educación sanitaria dirigido a las mujeres trabajadoras. Los temas desarrollados fueron: medicina preventiva, alimentación y nutrición, control de embarazo y planificación familiar. ${ }^{32}$ Hasta bien entrados los años 80, las actividades de las mujeres en las asociaciones de vecinos y en los centros de la mujer en relación con el derecho y la accesibilidad a la anticoncepción fueron frecuentes en Murcia. ${ }^{33}$

\section{EL ACTIVISMO POR LA PLANIFICACIÓN FAMILIAR EN MURCIA Y LAS PRIMERAS CONSULTAS PÚBLICAS, 1976-1980}

La primera consulta pública de planificación familiar en Murcia se estableció en el año 1976, antes de la despenalización de los anticonceptivos, en el hospital Virgen de la Arrixaca, bajo la asistencia del doctor Marín Mussó. Al igual que en Barcelona (1971), Madrid (1972), Granada (1975), Valladolid (1976) y Sevilla (1978), ${ }^{34}$ esta consulta se puso en marcha en un hospital público por iniciativa de algunos ginecólogos, en este caso varones: «Pues sí, éramos pioneros, pero ya te digo, la presión feminista, por así decirlo, vino después». ${ }^{35}$ José María Marín había tenido contacto y experiencias previas con la anticoncepción trabajando en Marruecos:

31 Línea, 30 de septiembre de 1980, p. 6.

32 «Ciclos de educación para mujeres», La Verdad, 24 de junio de 1977, p. 12.

33 Por ejemplo, en 1979: «Charla sobre orientación familiar en Molina», La Verdad, 9 de febrero de 1979, p. 15. «Charlas sanitarias en barriada Virgen de la Caridad», La Verdad, 28 de abril de 1979, p. 9; celebrada en Villanueva una charla-coloquio sobre planificación familiar, Linea (Cartagena), 20 de mayo de 1979, p. 17. Para la presencia de mujeres en el movimiento vecinal en Murcia, v. ALEMÁN PÉREZ, S., 2006.

34 RODRIGUEZ-OCAÑA, E.; IGNACIUK, Á. y ORTIZ-GÓMEZ, T.: «Ovulostáticos y Anticonceptivos. El Conocimiento Médico sobre la Píldora en España durante el Franquismo y la Transición Democrática (1940-1979)», Dynamis, 32, n. 2, 2012, pp. 467-494. ORTIZ GÓMEZ, T.; RODRIGUEZ OCAÑA, E. y GIL, E., 2013.

35 José María Marín Mussó, ginecólogo, entrevistado por Ramón Castejón, Murcia, 24 de noviembre de 2014. 
«Y luego me vine a España, me fui a Lorca, y de Lorca concursé y me vine a la Arrixaca. Y aquí abrimos, inmediatamente que llegamos, educación maternal, y al mismo tiempo, regulación de natalidad, que nosotros le llamábamos así. Le llamábamos así por razones óbvias. Porque en aquel tiempo todavía la cosa no estaba del todo bien a pesar que estábamos en el 76 [...] pero tampoco nos impedía hacer nada. Esa es la verdad. O sea, nosotros jamás tuvimos inconveniente ninguno». 36

La consulta contó desde el principio con el estímulo y apoyo del Jefe de Servicio en el hospital y catedrático de Obstetricia y Ginecología en la Facultad de Medicina de Murcia, doctor Lorenzo Abad Martínez Martínez. ${ }^{37}$ La posición moral y social sobre el uso de anticonceptivos de este médico había cambiado desde su postura inicial en la década de los sesenta en la que defendía la «indicación terapéutica» de estos fármacos, por ser la única moralmente aceptable. ${ }^{38}$ Como otras consultas en los hospitales públicos, en la segunda mitad de los setenta tuvo una gran aceptación: «Pero es que teníamos una consulta masiva. O sea, de 70-80 señoras cada día». Contrastando con esta actividad, no hay ninguna referencia a esta consulta en la prensa murciana antes de la despenalización de los anticonceptivos, lo que refuerza su carácter «clandestino». En consonancia con las tendencias en la prensa estatal durante el periodo 19751978, ${ }^{39}$ aunque más claramente en el año 1978 -coincidiendo con su despenalización-, la prensa murciana reflejaba un ambiente más abierto y tolerante en relación con la planificación familiar. De hecho, a partir de 1977 el

36 Ibídem. La consulta venía recogida en el informe que elaboró la Federación Internacional de Planificación Familiar (Región Europea) sobre los centros de PF en España en 1982. En el informe aparecía como consulta de ginecología coordinada con diagnóstico precoz del cáncer y se hacía referencia a que ofrecía asistencia desde hacía muchos años. FEDERACIÓN INTERNACIONAL DE PLANIFICACIÓN FAMILIAR: Centros de planificación familiar en España, 1982, s.1., s.e., s.a.

37 Lorenzo Abad Martínez (1941-2013) se incorporó como profesor de Obstetricia y Ginecología a la Facultad de Medicina de Murcia en 1972, procedente de la Universidad de Valencia, y en 1975 accede a la cátedra. Sobre su producción científica en Murcia, v. MARSET CAMPOS, P. y SÁEZ GÓMEZ, J. M.: «La ciencia médica en la segunda parte del Franquismo. Los inicios de la Facultad de Medicina de Murcia (1968-1976)», en P. Marset Campos; J. M. Sáez Gómez y C. López Fernández (dirs.): Ciencia e Instituciones Cientificas en la Región de Murcia. El Franquismo (1936-1975), Murcia: Universidad de Murcia, 2013. La Fundación Española de Contracepción le dedicó un homenaje en 2013.

38 ABAD MARTÍNEZ, L.: «Las nuevas drogas anticoncepcionales. Puntos de vista deontológicos», Revista Española de Obstetricia y Ginecología, 21, n. 137, 1964, pp. 317-326. Para una revisión de los aspectos morales y sociales del uso de los anovulatorios en la prensa médica española, véase: IGNACIUK, Á. y ORTIZ GÓMEZ, T.: Anticoncepción, mujeres y género. La píldora en España y Polonia (1960-1980), Madrid: Los Libros de la Catarata, 2016, pp. 89-106.

39 ORTIZ-GÓMEZ, T. \& IGNACIUK, Á.: «Pregnancy and labour cause more deaths than oral contraceptives: The debate on the pill in the Spanish press in the 1960s and 1970s», Public Understanding of Science, 24, n. 6, 2015, pp. 658-671. 
periódico Línea va modificando su discurso en relación con el tema de la planificación familiar. ${ }^{40}$ Sin embargo, La Verdad continuaba dando soporte a la corriente de opinión opuesta a los anticonceptivos 41 e introducía la oposición al aborto, utilizando el ejemplo francés y su ley de despenalización de 1976, posicionándose de cara al futuro debate en la prensa murciana. ${ }^{42}$

En la consulta del hospital participó Consuelo Gómez Eguilegor -que sería una ginecóloga pionera de la planificación familiar en Murcia-, como estudiante de Medicina y alumna interna colaborando con el dr. Marín Mussó en temas de anticoncepción y sexualidad. Posteriormente realizaría la especialidad de Ginecología y Obstetricia (MIR) en Cartagena. ${ }^{43}$ También tuvieron contacto con esta consulta Marisa Blanco, médica, militante comunista y posteriormente presidenta de la Asociación Murciana de Planificación Familiar (AMPF) (1978-1979), y Carmen Castro, médica que se vinculó posteriormente a los servicios de planificación familiar de la Consejería de Sanidad. La consulta de regulación de la natalidad del hospital Virgen de la Arrixaca se constituyó desde 1976 en el espacio de formación y difusión de la práctica en planificación familiar de la provincia.

La doctora Marisa Blanco participó activamente en el movimiento por la planificación familiar en Murcia. Junto a otras militantes comunistas y profesionales sanitarios tuvo un papel fundamental en la constitución de la AMPF. El 12 de mayo de 1978, en el local del Colegio de Médicos de Murcia, tuvo lugar una asamblea para la creación de esta asociación. ${ }^{44}$ El 5 de octubre se aprobaban sus estatutos y el 19 se celebraba en el Colegio de Médicos de Murcia una reunión para definir los objetivos, el programa y elegir la junta directiva. Marisa Blanco fue elegida presidente de la asociación en esa reunión.

40 Por ejemplo, en junio, Línea se hacía eco del Seminario de Planificación Familiar realizado en Mahón y organizado por el doctor Luis Cañada Royo: «España no puede permanecer ajena a la planificación familiar», Línea, 25 de junio de 1977, p. 15. Otras noticias y artículos en 1977 favorables: «El Consejo de Mujeres de la Internacional Socialista pide la despenalización de la anticoncepción y el aborto», Línea, 15 de octubre de 1977, p. 32. «Píldora anticonceptiva», Línea, 30 de diciembre de 1977, p. 16.

41 «Contraceptivos y mediocridad», La Verdad, 27 de diciembre de 1977, p. 20. Artículo firmado por Isidoro Valverde Álvarez (1929-1995), miembro del Cuerpo Jurídico de la Armada, escritor y periodista, fue cronista oficial de Cartagena en dos ocasiones y obtuvo una diplomatura en orientación familiar por el Instituto de Ciencias de la Educación de la Universidad de Navarra en 1975.

42 «El derecho a nacer», La Verdad, 16 de diciembre de 1977, p. 4.

43 Consuelo Gómez Eguilegor, ginecóloga y pionera de la Planificación Familiar en Murcia, entrevistada por Ramón Castejón, Cieza, 2 de junio de 2014. Empezó la residencia de la especialidad de Ginecología y Obstetricia en el hospital Virgen del Rosell de Cartagena en 1978. A través de una vía de acceso al título de especialista médico anterior al sistema MIR, consiguió la especialidad antes de finalizar el periodo de formación de MIR.

44 «Asociación de Planificación Familiar», Línea, 12 de mayo de 1978, p. 2. «Gestiones para crear la Asociación de Planificación Familiar», La Verdad, 12 de mayo de 1978, p. 3. 
El primer objetivo era, según la prensa, «ofrecer la máxima información sexual posible, incidiendo en las clases sociales menos favorecidas culturalmente». La asociación pretendía promover y asesorar la creación de centros de planificación familiar en centros de salud, ambulatorios y hospitales, informar sobre la prestación de servicios anticonceptivos, promover cambios de actitudes sociales con respecto a la planificación familiar y trabajar por la modificación de la situación sanitaria y social en relación con ella. Durante la reunión se discutió el programa de trabajo de la asociación y se constituyó la junta directiva. Junto a Marisa Blanco se eligió como vicepresidente a José María Marín Mussó ${ }^{45}$ y como secretaria a Regina Urtibi Sáez. Una tesorera y nueve vocales, cinco mujeres y cuatro hombres, completaban la junta directiva. ${ }^{46}$ La asociación informaba pocos días después del buen recibimiento que la iniciativa asociacionista había tenido en los medios médicos especializados y en concreto el apoyo que había recibido del Servicio de Obstetricia y Ginecología del hospital Virgen de La Arrixaca. Igualmente informaba del apoyo recibido por parte de la Federación de Asociaciones de Vecinos. ${ }^{47}$

La asociación ejemplarizaba en Murcia, como otras asociaciones regionales de PF, la confluencia de intereses científico-médicos, políticos y sociales que se daban en el activismo por la planificación familiar. En este caso, no se identificó con el feminismo, sino con un activismo pro-derechos humanos y su actividad se dedicó fundamentalmente a dar charlas en colegios y en asociaciones de mujeres para promover y difundir los contenidos de la planificación familiar. La actividad de la asociación fue languideciendo y terminó desapareciendo en 1979.48 Por su parte, la Asociación Española de PF, en la que la asociación murciana se encontraba integrada, desarrolló su actividad entre 1977 y 1979. Supuso un intento de institucionalización que no llegó a consolidarse y que puso en evidencia la diversidad de intereses existente en el movimiento. ${ }^{49}$

45 El dr. Marín Mussó había participado en la reunión constitutiva de la Federación de asociaciones de Planificación Familiar que tuvo lugar en Zaragoza los días 11 y 12 de febrero de 1978. Para la reunión de Zaragoza, v. MIQUEO, C.: «Azar y necesidad: el movimiento por la planificación familiar y la libertad sexual en Zaragoza», en R. Campos Marín; Á. González de Pablo; M. I. Porras Gallo y L. Montiel: Medicina y poder político. XVI Congreso SEHM, pp. 247-252.

46 «Asociación Murciana de Planificación Familia. Dará la máxima información sexual posible al pueblo», La Verdad, 21 de octubre de 1978, p. 5.

47 Dicen los organizadores: Caluroso apoyo médico a la Asociación Murciana de Planificación Familiar. Línea, 22 de octubre de 1978, p. 10.

48 María Isabel Blanco Marín, médica, militante comunista, activista por la planificación familiar, entrevistada por Ramón Castejón, Murcia, 29 de enero de 2015.

49 ORTIZ GÓMEZ, T.; FAJARDO, A.; GIL GARCÍA, E.; IGNACIUK, Á. y RODRÍGUEZ OCAÑA, E., 2011. 
A la consulta en el hospital Virgen de la Arrixaca siguió, una vez despenalizados los anticonceptivos en octubre de 1978, la apertura del primer centro de orientación familiar extrahospitalario en Murcia. Fue abierto a finales de 1978 por el Ministerio de Sanidad y Seguridad Social en la Delegación provincial de Sanidad dentro de su plan de creación de centros piloto. ${ }^{50}$ Hay que señalar que este centro no estuvo dotado económicamente hasta finales de 1979. Según reflejaba la prensa el personal no cobró retribuciones durante todo el periodo que estuvo abierto. ${ }^{51}$ La importancia del PCE dentro de las fuerzas políticas murcianas y del activismo por la planificación familiar en el inicio de este primer servicio de planificación familiar quedaba representado por Marisa Blanco, presidenta de la AMPF. Había finalizado los estudios de Medicina ese mismo año y, sin embargo, participó en su organización y se incorporó a él desde su apertura en diciembre de 1978.

En efecto, el 28 de noviembre se reunían en la Dirección provincial de Sanidad el maternólogo del estado Cortés Gil, Marisa Blanco, en calidad de presidenta de la AMPF, Elvira Ramos, jefa del servicio de Epidemiología de la Dirección provincial de Salud y militante comunista, Gloria Fuentes, trabajadora social, y una matrona. El centro contó pues con dos activistas por la planificación familiar y militantes comunistas. La reunión tenía como objetivo definir el funcionamiento y la organización del centro que se iba a abrir próximamente. Cortés Gil y Marisa Blanco asistirían en Madrid los días 11 y 12 de diciembre a una reunión de coordinación de los 16 centros pilotos que el Ministerio iba a abrir en breve. El centro se iba a dedicar, según la nota publicada en la prensa, a la planificación y al consejo genético de las familias que lo desearan. ${ }^{52}$

El Centro de Orientación Familiar de Sanidad, como era conocido, funcionó desde el 14 de diciembre de 1978 hasta el 10 de febrero de 1980. En ese periodo se realizaron 1.566 consultas de mujeres y parejas jóvenes. A los dos o tres meses

50 Real Decreto 2275/1978, de 1 de septiembre, sobre establecimientos de servicios de orientación familiar, BOE de 25 de septiembre de 1978, n. 229, p. 22333.

51 Pregunta socialista al Gobierno sobre el cierre del Centro Oficial de Orientación Familiar, Línea, 21 de marzo de 1980, p. 5.

52 Instalado en la Delegación de Sanidad. El día 14 entrará en funcionamiento el primer centro de planificación familiar, Línea, 30 de noviembre de 1978, p. 4. Elvira Ramos había recibido formación sobre planificación familiar en Londres en 1969 en un curso de la International Planned Parenthood Federation. Asunción Villatoro, una médica pionera de la planificación familiar en España, haría un curso similar en 1970 con la misma organización. Curriculum Vitae (2003) cedido por Elvira Ramos García. ORTIZ-GÓMEZ, T. \& IGNACIUK, Á., 2018. Para una aproximación a la relación entre las activistas por la anticoncepción en España y el asociacionismo internacional de PF, v. ORTIZ GÓMEZ, T.; FAJARDO, A.; GIL GARCÍA, E.; IGNACIUK, Á. y RODRÍGUEZ OCAÑA, E., 2011. 
de la apertura del centro, la ginecóloga Consuelo Gómez Eguilegor ${ }^{53}$ se incorporó al equipo médico inicial constituido por el maternólogo Cortés y la doctora Marisa Blanco. Estuvo atendido durante su existencia por dos médicas, una trabajadora social, Carmen Alburquerque, y una matrona.

El centro abierto en Murcia formaba parte de los 15-16 que abrió el Ministerio en Santander, Sevilla, Valencia, Valladolid, Zaragoza, Baleares, Barcelona, La Coruña, Granada, Madrid, Navarra, Salamanca, y Tenerife. Al día siguiente de la apertura no había acudido nadie al centro, lo que contrasta con la demanda que tenía la consulta de regulación de la natalidad del hospital Virgen de La Arrixaca y con los relatos de otras protagonistas de los primeros centros de PF en España. ${ }^{54} \mathrm{El}$ centro era gratuito y funcionaba todas las tardes de cuatro a nueve de la tarde. Para dar a conocer el centro y aumentar la demanda empezaron a organizarse unas charlas en la Dirección provincial y posteriormente fuera de ella, a cargo de los médicos. Se celebraban en salones de los ayuntamientos, salones parroquiales y locales de diferentes asociaciones. Las demandaban asociaciones de vecinos, de padres de alumnos e incluso algunas asociaciones de amas de casa, como la de la Alberca, en el marco de la VI semana de la mujer. ${ }^{55}$

En el año 1979, la representación en la prensa murciana de los debates parlamentarios referentes a la planificación familiar se centra en dos aspectos: el debate sobre las políticas reproductivas del estado y el cumplimiento del decreto para el establecimiento de Centros de Orientación Familiar de septiembre de 1978, fundamentalmente en los aspectos de dotación presupuestaria y su inclusión dentro de la reforma sanitaria en curso. El senador del PSOE Delgado Ruiz interpelaba al Gobierno de la UCD en el Pleno del Senado de 12 de junio acerca del incumplimiento de dicho decreto. El ministro de Sanidad, Rovira Tarazona, respondía afirmando que la planificación familiar no era tarea del estado, que éste debía respetar la libertad del individuo y la pareja: «El Estado debe asegurar esa

53 Ángel Fernández Nafria, delegado territorial del Ministerio de Sanidad en Murcia, y Enrique Viviente López, director provincial de Sanidad entrevistaron a Consuelo Gómez para su incorporación al centro. Su interés era incorporar a una especialista en Ginecología: «Entonces pues necesitaban que alguien fuera, creo que era, creo recordar que era un par de veces a la semana, y necesitaban un ginecólogo, porque iban a montar una consulta de planificación familiar en condiciones porque hasta entonces lo único que había era la dispensación de píldoras anticonceptivas, pero querían, sobre todo por los DIU». Entrevistada por Ramón Castejón, Cieza, 2 de junio de 2014.

54 ORTIZ-GÓMEZ, T. \& IGNACIUK, Á., 2018.

55 «Mañana comienza la VI Semana de la Mujer en La Alberca», La Verdad, 6 de mayo de 1979, p. 16. «El lunes comienza la I Semana de Cultura Popular en Beniaján», La Verdad, 6 de octubre de 1979, p. 13. A las 8 de la noche, en la Casa del Pueblo, calle Cuatro Santos... charla-coloquio sobre el tema «Anticonceptivos y planificación familiar», a cargo de Marisa Blanco, Línea (Cartagena), 21 de noviembre de 1979, p. 12. La mayor parte de estas charlas las daba efectivamente Marisa Blanco. 
libertad, pero debe contribuir a su orientación y formación en materia de salud sexual y planificación familiar».

Era pues un discurso ambivalente que, por una parte, se distanciaba de hacer suya una política reproductiva estatal de control de nacimientos que había recibido críticas de los sectores católicos y de la derecha franquista, y, por otra, asumía el nuevo rol del estado en las políticas del salud sexual y reproductiva. Se asistía, por tanto, a un cambio en relación con las políticas pronatalistas del franquismo -que se habían sustentado en prohibiciones legales relacionadas con el aborto y la anticoncepción y el apoyo limitado a las madres y a las familias-, y la irrupción de una nueva política sexual por parte del estado democrático, que asumía la planificación familiar dentro del sistema sanitario ${ }^{56}$ y cierta responsabilidad en la salud sexual de sus ciudadanos. El ministro planteó también la cuestión de la ubicación de estos centros, que no podían considerarse, «aisladamente de lo que debe entenderse como medicina comunitaria y primera medicina» y que por ello, el tema se incluiría en el documento de reforma sanitaria a presentar ese mes de junio en el Congreso. La intervención del senador Vallejo, de Socialistas de Andalucía, introdujo la cuestión del papel de las autonomías en el tema, cuestión que sería de crucial importancia en la expansión de los CPF en Murcia. ${ }^{57}$

El Centro de Orientación Familiar de Sanidad, al igual que el resto de centros que formaban parte del programa del Ministerio, nació sin presupuesto y no había remuneración económica para los profesionales que lo atendían. ${ }^{58}$ Sin embargo, continuó su actividad a pesar de no tener financiación - de hecho los directores de los centros habían establecido un ultimátum conjunto al ministerio exigiéndola y amenazando con cerrar el 1 de noviembre de 1979 si no la recibían-, y prestando servicios de planificación familiar, a los que se unieron la detección del cáncer genital femenino, con una creciente demanda por parte de las mujeres.

A pesar de estas condiciones adversas, el entusiasmo y voluntarismo de sus profesionales, todas ellas mujeres si exceptuamos al maternólogo del estado que les permitió hacer sin cortapisas, ${ }^{59}$ posibilitó el desarrollo de ciertas actividades hasta su cierre en febrero de 1980. En este centro se desarrolló un estilo de trabajo y una relación menos jerarquizada entre las profesionales y las mujeres, carac-

56 IGNACIUK, Á., 2015, p. 95. ORTIZ GÓMEZ, T.; FAJARDO, A.; GIL GARCÍA, E.; IGNACIUK, Á. y RODRÍGUEZ OCAÑA, E., 2011.

57 «La planificación familiar no es tarea del Estado», Línea, 13 de junio de 1979, p. 24. «La planificación familiar se incluirá en la reforma sanitaria», La Verdad, 13 de junio de 1979, p. 40.

58 Entró en funcionamiento el primer centro de planificación familiar. Línea, 15 de diciembre de 1978, p. 7.

59 Elvira Ramos García, médica, especialista en Salud Pública, militante comunista, entrevistada por Ramón Castejón, Murcia, 4 de mayo de 2016. 
terísticas que posteriormente se trasladarían a los Centros Asesores de la Mujer y la Familia del Consejo Regional, ${ }^{60}$ dado que estas profesionales se incorporaron a ellos. Así, la doctora Marisa Blanco se incorporó al Centro Asesor abierto en junio de 1980 en la Plaza Preciosa de Murcia y la ginecóloga Consuelo Gómez lo haría poco después.

\section{CONCLUSIONES}

En Murcia, igual que en otras grandes ciudades españolas, la primera consulta de planificación familiar se puso en marcha en un hospital público antes de la legalización de los anticonceptivos en 1978. Desde 1976 en el hospital Virgen de la Arrixaca funcionaba una consulta de regulación de la natalidad que posibilitó el acceso a la anticoncepción a determinadas mujeres. Se confirma, como ocurrió en el resto del país, que el activismo por la planificación familiar se inició en Murcia de la mano de sectores médicos liberales. Esta consulta del hospital Virgen de la Arrixaca constituyó desde 1976 el espacio de formación y difusión de la práctica en planificación familiar de la provincia. Posteriormente, el movimiento por la planificación familiar en Murcia se estructuró entre 1978-1979 en torno a la AMPF, liderada por militantes comunistas en confluencia con intereses científico-médicos. Tras la legalización en 1978, en la Dirección provincial de Sanidad se abrió un centro de orientación familiar dentro del plan de establecimiento de estos centros, que el Ministerio de Sanidad del gobierno de la UCD dispuso ese mismo año. El centro no contó con dotación presupuestaria, pero desarrolló su actividad entre 1978 y 1980 sobre la base de la implicación profesional y política de su personal, buena parte de ellas activistas por la planificación familiar. A partir de 1978, el movimiento feminista en Murcia, organizado de manera autónoma en la Asamblea de Mujeres de Murcia, incluyó en su agenda el acceso a la anticoncepción, el derecho al aborto «libre y gratuito» y la sexualidad de las mujeres. Como consecuencia, el movimiento feminista, junto a otros actores políticos y sociales, tendría posteriormente un protagonismo central en la demanda y obtención de la apertura desde 1980 de los Centros Asesores de la Mujer y la Familia por parte del Consejo Regional de Murcia, en los que se incluiría la planificación familiar.

60 Algunas autoras consideran que estas nuevas relaciones entre profesionales y mujeres habrían tenido su origen en los primeros centros de planificación familiar de marcado carácter feminista y habrían sido desarrollados por médicas feministas. ORTIZ GÓMEZ, T. \& IGNACIUK, A., 2018. 


\section{Agradecimientos}

Esta investigación no habría podido realizarse sin la generosa colaboración de las personas entrevistadas. A todas ellas mi más sincero agradecimiento por dedicarme tiempo y por ceder parte de su memoria. Agradezco a Juana Saura Arnau su ayuda para llegar a varias de las protagonistas del activismo feminista y por la planificación familiar en Murcia. Gracias también al personal del Archivo Municipal de Murcia que hizo el trabajo más fácil, atendiendo con amabilidad mis búsquedas. Juana Vera Díaz revisó el manuscrito mejorando la versión final y a ella se debe que la investigación viera la luz. Finalmente, muchas gracias a los colegas del proyecto ASYS y a Patricia Navarro por su trabajo de transcripción. 